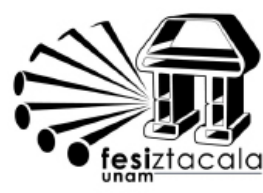

Revista Mexicana de Trastornos Alimentarios

Mexican Journal of Eating Disorders

Journal homepage: http://journals.iztacala.unam.mx/

Family Eating Disorders antecedent and symptomatology in women with an Eating Disorder (ED)

Antecedentes familiares y sintomatología en mujeres con Trastorno de la Conducta Alimentaria

Rodrigo César León Hernández, Araceli Aizpuru de la Portilla

Fundación mexicana contra la anorexia y la bulimia "Ellen West"

Abstract

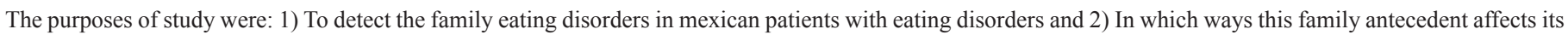

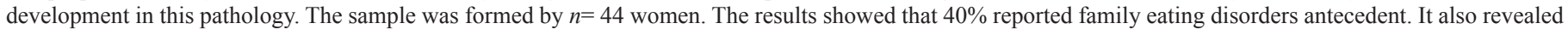

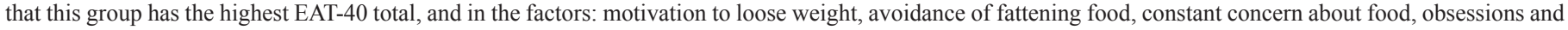

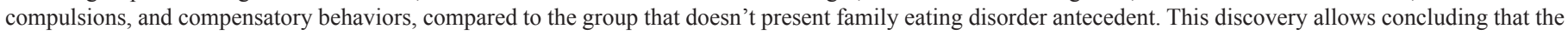
magnitude of the symptomatology is bigger whit the presence oh this antecedent.

\title{
Resumen
}

Los objetivos fueron: 1) detectar la presencia de antecedentes familiares de sintomatología alimentaria en una muestra de pacientes mexicanas con trastorno alimentario, y 2) de que forma influyen dichos antecedentes en la patología de este trastorno. La muestra estuvo formada por $n=44$ mujeres. Los resultados mostraron que el $40 \%$ reportó antecedentes de trastorno alimentario en la familia. Fue en este mismo grupo que se detectó mayor puntaje en el total del EAT-40 así como en los factores: motivación para adelgazar, evitación de alimentos engordantes, preocupación por la comida, obsesiones y compulsiones, y conductas compensatorias, al compararlo con el grupo sin antecedentes familiares de trastorno alimentario. Este hallazgo permite concluir que la magnitud de la sintomatología es mayor cuando existen antecedentes entre la familia.

\section{INFORMACIÓN ARTÍCULO}

Recibido 06/10/2010

Revisado 14/10/2010

Aceptado 02/12/2010

\section{Introducción}

Los Trastornos de la Conducta Alimentaria (TCA), al igual que la mayoría de las enfermedades que aquejan a nuestra sociedad, derivan de una etiología multicausal (bio-psico-socio-cultural). Sin embargo, el papel de la familia adquiere especial relevancia ya que representa el núcleo primario en el cual se transmiten normas, roles, costumbres y hábitos que influyen en estilos de vida, y más aún en los estilos y actitudes alimentarias. Por tal motivo, Trojovsky et al., (1995) consideran específicamente al medio familiar como responsable y sostén de estos trastornos.

Existe una estrecha relación entre disfunción familiar y TCA, una de las primeras aproximaciones fue realizada por Minuchin, Rosman y Baker (1978),

Correspondencia: Carretera México Toluca No 3847 Km. 20.5 Cuajimalpa de Morelos, C. P. 05000. Teléfono: 58120877. Correo electrónico: investigacion@ellenwest.org, araceliaizpuru@prodigy.net.mx quienes definieron a las familias con una integrante anoréxica como familias psicosomáticas. Los estudios posteriores reflejan una diversidad de patrones de interacción en el seno de las familias con un integrante que padece trastorno alimentario (Toro, 2004; Yager, 1982), por tanto y en coincidencia con Espina, Pumar, García y Ayerbe (1995), se puede afirmar que no existe un solo tipo de interacción o un modelo de funcionamiento familiar particular en estos trastornos.

Otra de las líneas de investigación la constituyen los estudios que han documentado antecedentes psiquiátricos en familias con TCA, al respecto Woodside, Shekter-Wolfson, Brandes y Lackstrom (1993) encontraron que entre los integrantes de estas familias existen antecedentes de trastornos afectivos, adicciones, trastornos alimentarios y obesidad. De igual forma, diversos autores han argumentado que en familias de pacientes con psicopatología bulímica 
existe mayor prevalencia de depresión, alcoholismo y abuso de drogas, en comparación con familias sin integrantes con TCA (Fairburn, Cooper, Doll \& Welch 1999; Moorhead et al., 2003; Schmidt, Humfress \& Treasure, 1997). Aunado a lo anterior Hudson, Pope, Jonas y Yurgelun-Todd (1983), detectaron que en familias de estos pacientes existen porcentajes mayores de trastornos afectivos cuando se comparan con familias con otros trastornos psiquiátricos tales como trastorno bipolar, esquizofrenia y trastorno límite de personalidad.

Ahora bien, con respecto a la transmisión generacional de la patología alimentaria, asunto de mayor interés en el presente escrito, los estudios pioneros en el tema indican que los padres de pacientes con TCA presentan problemas de peso y preocupación por la comida (Crisp, Harding \& McGuiness, 1974). Específicamente, los padres de pacientes con anorexia presentan mayor insatisfacción con su cuerpo, preocupación por el peso y seguimiento de dietas restringidas (Kalucy, Crisp \& Harding, 1977).

Son distintas las maneras en que el entorno familiar puede contribuir al desarrollo de estos padecimientos, Thelen y Cornier (1995) fueron pioneros en sugerir que la presión de padres a hijas, para controlar el peso, se asocia al desarrollo de sintomatología de TCA desde muy temprana edad (9 y 10 años). Confirmando lo anterior, Edmund y Hill (1999) documentaron que los niños y niñas que hacen dieta alrededor de los 12 años, en su mayoría reportan estar bajo el control alimentario de sus padres.

El papel de la influencia familiar en la transmisión de las conductas de riesgo podría explicarse por la transmisión de normas, aprendizaje social o modelado de las propias actitudes de los padres. Toro (2004) resalta el hecho de que a muy corta edad, los niños se conducen alimentariamente de una manera muy similar a los padres. Al respecto, Jacobi, Agras y Harmmer (2001) siguiendo niños desde su nacimiento hasta los 8 años, encontraron que aquellas madres que restringían su alimentación predecían la preocupación por la gordura en sus hijas.

Otro de los roles de suma importancia en la transmisión familiar es el papel de la presión social ha- cia la delgadez. En un estudio realizado por Levine, Smolak, Moodey, Shuman y Hessen (1994) se encontró que las desaprobaciones y la crítica por parte de la familia, en relación al peso, predicen los problemas alimentarios en las hijas adolescentes. De manera similar, se ha documentado que las burlas o bromas tanto de familiares como de amigos o compañeros, se asocian con insatisfacción corporal, baja autoestima, síntomas depresivos e intentos suicidas (Eisenberg, Neumark-Sztainer \& Story, 2003).

Los antecedentes descritos con anterioridad sugieren que existen distintas vías por las cuales se presenta la transmisión generacional en el seno familiar. Al respecto, en un estudio longitudinal desarrollado por Moorhead et al. (2003), se encontró que lo que diferenciaba a las mujeres con TCA de aquellas que no lo desarrollaron fueron los antecedentes de estos trastornos en ambos padres o hermanos.

Con base en los estudios hasta aquí expuestos, los objetivos del presente estudio fueron: 1) explorar la existencia de antecedentes familiares de sintomatología alimentaria en una muestra de pacientes mexicanas con trastorno de la conducta alimentaria, y 2) de qué forma influyen dichos antecedentes en la patología de TCA.

\section{Método}

\section{Participantes}

La muestra no probabilística estuvo formada por $n=44$ pacientes diagnosticados de TCA (Anorexia, Bulimia o Trastorno Alimentario No Específico), con edad promedio $M E=19.7$ y $D E=6.01$. Del total de la muestra el 96\% fueron mujeres. El diseño del estudio fue transversal.

\section{Procedimiento}

Se realizó una evaluación diagnóstica, a todas aquellas personas que solicitaron tratamiento en la Fundación Ellen West en el periodo de enero a junio de 2010. La evaluación se realiza mediante una batería diagnóstica y una entrevista. La entrevista diagnóstica se aplica por el personal del área de psicología 
de dicha institución, además se complementa de la valoración nutricia y psiquiátrica. La recolección y el manejo de datos se hicieron de acuerdo a los criterios éticos que rigen a la psicología.

\section{Instrumentos}

La entrevista esta basada en criterios DSM-IV-TR para el diagnóstico de TCA, esta contiene un apartado que explora antecedentes familiares del trastorno. Dicha entrevista esta en proceso de validación.

La batería de pruebas estuvo integrada por el Inventario de Desórdenes Alimentarios, Test de Bulimia, Cuestionario de Imagen Corporal y el Test de Actitudes Alimentarias (por sus siglas en inglés EDI, BULIT, BSQ y EAT-40 respectivamente). Para propósitos del presente estudio se reportan únicamente los resultados del EAT-40 (Garner \& Garfinkel, 1979) en su versión adaptada y validada en población mexicana (Álvarez, Vázquez, Mancilla \& Gómez-Peresmitré, 2002). La prueba tiene buena consistencia interna $(\alpha=0.83)$ y esta compuesta por siete factores (1. Motivación para adelgazar, 2. Evitación de alimentos engordantes, 3. Preocupación por la comida, 4. Presión social percibida, 5. Obsesiones y compulsiones, 6. Patrones y estilos alimentarios alimentarios estereotipados y 7. Conductas compensatorias) que explican el 37.2\% de la variabilidad total. El criterio de calificación es a mayor puntaje mayor problema.

\section{Resultados}

Los análisis estadísticos se realizaron con el paquete estadístico para las ciencias sociales (SPSS-15). Del total de la muestra el $40 \%$ reportó antecedentes de TCA en familia. Las pacientes reportaron desde presencia de un TCA hasta control alimentario y preocupación por el peso y la silueta. Como se puede observar en la Figura 1, los porcentajes mayores se ubicaron en miembros de familia extensa (primas $12 \%$ y tías $10.5 \%$ ). Sin embargo, el control alimentario y la preocupación por el peso y la silueta fue mayor $(9.5 \%)$ en la familia de primer grado (sumatoria madre, padre y hermanos/as).

La distribución por diagnóstico fue: anorexia nervosa $38.5 \%$, bulimia nervosa $32.7 \%$ y TANE $28.8 \%$. Con el propósito de explorar si existían diferencias entre las actitudes alimentarias de las tres entidades diagnósticas, se aplico un Análisis de Varianza (ANOVA One-Way). Los resultados no mostraron diferencias en el puntaje total del EAT-40 entre los tres grupos (anorexia, bulimia y TANE). En la comparación por factores, únicamente se detectaron diferencias significativas en los factores IV "Presión social percibida" $F(2,42)=5.691, p=.006$ y VII "Conductas compensatorias" $F(2,42)=3.471, p=.039$ (ver Tabla 1). El análisis Post Hoc Bonferroni identificó diferencias entre anorexia y bulimia en el factor IV $(p=.008)$ y entre bulimia y TANE en el factor VII $(p=.036)$. Considerando que estas diferencias sólo se

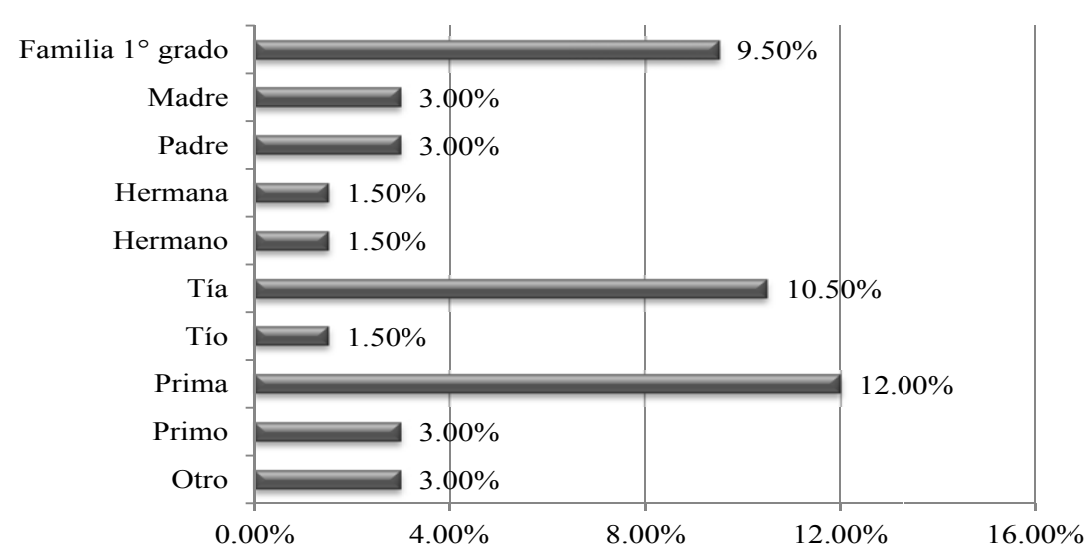

Figura I. Distribución porcentual de antecedentes de TCA en la familia. 
Tabla 1. ANOVA (One-Way). Comparación entre las tres entidades diagnósticas (Anorexia, Bulimia y TANE).

\begin{tabular}{|c|c|c|c|c|c|c|}
\hline EAT -40 & & $\begin{array}{c}\text { Suma de } \\
\text { cuadrados }\end{array}$ & $g l$ & $\begin{array}{c}\text { Media } \\
\text { cuadrática }\end{array}$ & $\boldsymbol{F}$ & $p$ \\
\hline \multirow{3}{*}{ Puntuación Total } & Entre grupos & 3631.91 & 2 & 1815.95 & 1.95 & .15 \\
\hline & Intra grupos & 43868.08 & 42 & 933.36 & & \\
\hline & Total & 47500.00 & 44 & & & \\
\hline \multirow{3}{*}{$\begin{array}{l}\text { FI. Motivación para } \\
\text { adelgazar }\end{array}$} & Entre grupos & 9.860 & 2 & 4.93 & .14 & .870 \\
\hline & Intra grupos & 1735.06 & 42 & 35.40 & & \\
\hline & Total & 1744.92 & 44 & & & \\
\hline \multirow{3}{*}{$\begin{array}{l}\text { F II. Evitación de } \\
\text { alimentos engordantes. }\end{array}$} & Entre grupos & 5.717 & 2 & 2.85 & .25 & .781 \\
\hline & Intra grupos & 564.28 & 42 & 11.51 & & \\
\hline & Total & 570.00 & 44 & & & \\
\hline \multirow{3}{*}{$\begin{array}{l}\text { F. III Preocupación por } \\
\text { la comida }\end{array}$} & Entre grupos & 9.83 & 2 & 4.91 & .27 & .765 \\
\hline & Intra grupos & 896.68 & 42 & 18.30 & & \\
\hline & Total & 906.51 & 44 & & & \\
\hline \multirow{3}{*}{$\begin{array}{l}\text { F. IV Presión social } \\
\text { percibida }\end{array}$} & Entre grupos & 102.31 & 2 & 51.15 & 5.70 & .006 \\
\hline & Intra grupos & 440.45 & 42 & 8.98 & & \\
\hline & Total & 542.76 & 44 & & & \\
\hline \multirow{3}{*}{$\begin{array}{l}\text { F. V Obsesiones y } \\
\text { compulsiones }\end{array}$} & Entre grupos & 4.50 & 2 & 2.25 & .23 & .792 \\
\hline & Intra grupos & 472.16 & 42 & 9.63 & & \\
\hline & Total & 476.73 & 44 & & & \\
\hline \multirow{3}{*}{$\begin{array}{l}\text { F. VI Patrones y estilos } \\
\text { alimentarios } \\
\text { estereotipados }\end{array}$} & Entre grupos & $9 ., 024$ & 2 & 45.512 & 2.81 & .070 \\
\hline & Intra grupos & 792.283 & 42 & 16.169 & & \\
\hline & Total & 883.308 & 44 & & & \\
\hline \multirow{3}{*}{$\begin{array}{l}\text { F. VII Conductas } \\
\text { compensatorias }\end{array}$} & Entre grupos & 69.602 & 2 & 34.801 & 3.471 & .039 \\
\hline & Intra grupos & 491.225 & 42 & 10.025 & & \\
\hline & Total & 560.827 & 44 & & & \\
\hline
\end{tabular}

presentaron en dos de los siete factores del EAT-40, se unificó la muestra incluyendo a las tres distintas entidades diagnósticas en un grupo (presencia de antecedentes familiares de TCA) para el análisis posterior.

En el análisis siguiente se aplicó una "t de Student para muestras independientes" con el propósito de explorar las diferencias en las actitudes alimentarias que explora el EAT-40, en "presencia versus ausencia" de antecedentes familiares de TCA. Se detectaron diferencias estadísticamente significativas en el puntaje total $[t(42)=2.449, p=.019] \mathrm{y}$ en los factores I $[t(42)=2.011, p=.049]$, II $[t(42)=2.258, p=.029]$, III $[t(42)=2.092, p=.043], \mathrm{V}[t(42)=2,842, p=.007]$ y VII $[t(42)=2.608, p=.013]$. La dirección de las medias indica que tanto en el total como en el puntaje por factores, el grupo con antecedentes de TCA en la familia presenta puntajes más altos, en comparación con el grupo sin dichos antecedentes (ver Tabla 2).

\section{Discusión y Conclusiones}

Entre los resultados se encontró que un porcentaje elevado (40\%) de los participantes refirió antecedentes de TCA en la familia; los porcentajes mayores se reportaron en familia extensa (primas y tías) y de primer grado (en el total de los integrantes). Estos datos confirman el fenómeno de la transmisión generacional en pacientes mexicanas y coinciden con la literatura internacional que ha documentado dicho fenómeno desde hace ya décadas atrás (Crisp et al. 1974; Kalucy et al. 1977; Moorhead et al. 2002). El porcentaje de pacientes que reportaron antecedentes familiares en el presente estudio es muy concordante con el reportado por Ochoa de Alda, Espina y Ortego (2006), quienes encontraron que el $43 \%$ de pacientes reporta antecedentes familiares de TCA.

El resultado de mayor relevancia fue el que se obtuvo al comparar las actitudes alimentarias entre 
Tabla 2. Comparación estadística ( $t$ de Student para muestras independientes) entre grupos: ausencia y presencia de Antecedentes Familiares de TCA.

\begin{tabular}{|c|c|c|c|c|c|}
\hline EAT-40 & $t$ & $g l$ & $p$ & $\begin{array}{c}\text { ME y DE } \\
\text { Grupo con } \\
\text { antecedentes } \\
\text { familiares }\end{array}$ & $\begin{array}{c}\text { ME y DE } \\
\text { Grupo sin } \\
\text { antecedentes } \\
\text { familiares }\end{array}$ \\
\hline Puntuación Total & 2.449 & 42 & .019 & $\begin{array}{l}M E=65.9 \\
D E=25.6\end{array}$ & $\begin{array}{l}M E=44.6 \\
D E=31.9\end{array}$ \\
\hline FI. Motivación para adelgazar & 2.011 & 42 & .049 & $\begin{array}{c}M E=10.3 \\
D E=4.7\end{array}$ & $\begin{aligned} M E & =7.0 \\
D E & =6.1\end{aligned}$ \\
\hline $\begin{array}{l}\text { F II. Evitación de alimentos } \\
\text { engordantes. }\end{array}$ & 2.258 & 42 & .029 & $\begin{aligned} M E & =5.4 \\
D E & =2.9\end{aligned}$ & $\begin{array}{l}M E=3.3 \\
D E=3.4\end{array}$ \\
\hline $\begin{array}{l}\text { F. III Preocupación por la } \\
\text { comida }\end{array}$ & 2.092 & 42 & .043 & $\begin{aligned} M E & =7.4 \\
D E & =3.5\end{aligned}$ & $\begin{array}{l}M E=4.9 \\
D E=4.1\end{array}$ \\
\hline F. IV Presión social percibida & 1.115 & 42 & .268 & $\begin{aligned} M E & =4.2 \\
D E & =3.2\end{aligned}$ & $\begin{array}{l}M E=3.0 \\
D E=3.4\end{array}$ \\
\hline F. V Obsesiones y compulsiones & 2.842 & 42 & .007 & $\begin{aligned} M E & =6.5 \\
D E & =1.2\end{aligned}$ & $\begin{array}{l}M E=4.2 \\
D E=3.4\end{array}$ \\
\hline $\begin{array}{l}\text { F. VI Patrones y estilos } \\
\text { alimentarios estereotipados }\end{array}$ & 1.346 & 42 & .189 & $\begin{array}{l}M E=6.2 \\
D E=4.3\end{array}$ & $\begin{array}{l}M E=4.5 \\
D E=4.0\end{array}$ \\
\hline $\begin{array}{l}\text { F. VII Conductas } \\
\text { compensatorias. }\end{array}$ & 2.608 & 42 & .013 & $\begin{aligned} M E & =4.2 \\
D E & =1.7\end{aligned}$ & $\begin{array}{l}M E=3.4 \\
D E=2.9\end{array}$ \\
\hline
\end{tabular}

pacientes con antecedentes familiares de TCA y pacientes que no reportaron estos antecedentes. Los análisis mostraron que tanto en el puntaje total del EAT-40 como en 5 de los factores que conforman la versión mexicana (motivación para adelgazar, evitación de alimentos engordantes, preocupación por la comida, obsesiones y compulsiones, y conductas compensatorias), el grupo de pacientes que reportaron la presencia de sintomatología de trastorno alimentario en la familia, presentó un puntaje mayor y estadísticamente significativo. Este hallazgo permite concluir que la magnitud de la sintomatología es mayor cuando existen antecedentes en el seno familiar. Por tanto, el deterioro de la salud puede ser mayor y más difícil su recuperación. Así también, confirma la necesidad de incluir la terapia familiar en el tratamiento integral de los TCA.

La complejidad del fenómeno de la transmisión familiar puede estar asociada a presiones y a la persuasión para obedecerlas (Toro, 2004), ejemplo de ello son los estudios citados con anterioridad, los cuales documentan algunos factores tales como el control alimentario y del peso corporal, la presencia de sintomatología en familia, así como la presión social hacia la delgadez, las burlas y las criticas referentes al peso, que se asocian comúnmente al desarrollo de sintomatología de TCA en hijos desde muy temprana edad (Edmund \& Hill, 1999; Fairburn, et al. 1999; Jacobi, et al. 2001; Levine et al. 1994; Thelen \& Cornier, 1995).

Una de las ventajas del presente estudio es que fue realizado en población clínica y no en población normal, de tal forma los resultados aportan elementos de gran importancia para el estudio de la transmisión generacional, fenómeno que es documentado ya en distintos países y que contribuye al incremento epidemiológico de los TCA. Así también, cabe señalar que existen limitaciones tales como no poder generalizar los resultados ya que la muestra se obtuvo de personas que acudieron a solicitar tratamiento y no sabemos cómo se comporte este fenómeno en la población que no esta recibiendo atención.

Por otra parte, los resultados derivados del presente estudio permiten sugerir que el fenómeno de la 
transmisión familiar se considere uno de los aspectos de mayor interés en el ámbito de investigación, tratamiento y prevención de los TCA. Si bien es cierto que se cuenta con diversos estudios antecedentes que dan luz a este fenómeno, también se puede afirmar que la ausencia de acciones dirigidas a empoderar el ámbito familiar con estrategias que promuevan la transmisión de un modelo estético corporal normopeso y conductas alimentarias saludables, podría traer como consecuencia, el incremento en los índices epidemiológicos de estos trastornos.

\section{Referencias}

Álvarez, R. G., Vázquez A. R., Mancilla D. J. M. \& Gómez-Peresmitré G. (2002). Evaluación de las propiedades psicométricas del Test de Actitudes Alimentarias (EAT-40) en mujeres mexicanas. Revista Mexicana de Psicología, 19, 47-56.

Crisp, A. H., Harding, B. \& McGuiness, B. (1974). Anorexia nervosa. Psychoneurotic characteristics of parents: relationship to prognosis. Journal of Psychosomatic Research, 18, 167-173.

Edmunds, H. \& Hill, A. J. (1999). Dieting and the family context of eating in Young adolescent children. International Journal of Eating Disorders, 24, 435-440.

Eisenberg, M. A., Neumark-Sztainer, D. \& Story, M. (2003). Association of weight-based teasing and emotional well-being among adolescents. Archives of Pediatrics and Adolescent Medicine, 157, 733-738.

Espina, A., Pumar, B., García, A. \& Ayerbe, A. (1995). Una revisión de los estudios controlados sobre interacción familiar en la anorexia nerviosa. Cuadernos de Terapia Familias, 27, 5-17.

Fairburn,C. G,, Cooper, Z., Doll, H.A. \& Welch, S. L. (1999). Risk factors for anorexia nervosa. Three integrated case-control comparisons. Archives of Pediatrics and Adolescent Medicine, 56, 468-476.

Hudson, J. L., Pope, H. G.; Jonas, J. M. \& Yurgelun-Todd, D. (1983). A family history study of anorexia nervosa and bulimia. British Journal of Psychiatry, 142, 133138.

Garner, D. M. \& Garfinkel, P. E. (1979). The Eating Attitudes Test: an index of the symptoms of anorexia ner- vosa. Psychological Medicine, 9, 273-279.

Jacobi, C., Agras, W. S. \& Harmmer, L. (2001). Predicting children's reported eating disturbances at 8 years of age. Journal of the American Academy of Child \& Adolescent Psychiatry, 40, 364-372.

Kalucy, R. S., Crisp, A. H. \& Harding, B. (1977). A study of 56 families with anorexia nervosa. British Journal of Medical Psychology, 50, 381-95.

Levine, M. P., Smolak, L., Moodey, A. F., Shuman, M. D. \& Hessen, L. D. (1994). Normative developmental challenges, dieting, and eating disturbances in middle school girls. International Journal of Eating Disorders, 15, 603-610.

Minuchin, S., Rosman, B. L. \& Baker, L. (1978). Psychosomatic families: Anorexia Nervosa in context. Cambridge: Harvard University Press.

Moorhead, D. J., Stashwick, C. K., Reinherz, H. Z., Giaconia, R. M., Striegel-Moore, R. \& Paradis, A. D. (2003). Child and adolescent predictors for eating disorders in a community population of young adult woman. International Journal of Eating Disorders, $33,1-9$.

Ochoa de Alda, I., Espina, A. \& Ortego, M. A. (2006). Un estudio sobre personalidad, ansiedad y depresión en padres de pacientes con un trastorno alimentario. Clinica y Salud, 17, 151-70.

Schmidt, U., Humfress, H. \& Treasure, J. (1997). The role of general family environment and sexual and physical abuse in the origins of eating disorders. European Eating Disorders Review, 5, 184-207.

Thelen, M. H. \& Cornier, J. F. (1995). Desire to be thinner and weight control among the children and their parents. Behavior Therapy, 26, 71-83.

Toro, J. (2004). Riesgo y causas de la anorexia nerviosa. Barcelona: Ariel.

Trojovsky, A., Scheer, P., Dunitz, M., Kaschnitz, W., Sommer, I. \& Kranz, U. (1995). Pubertätsmagersucht (Anorexia nervosa). Paediatrie und Paedologie, 30, 93-99.

Woodside, D. B., Shekter-Wolfson, L. F., Brandes, J. S. \& Lackstrom, J. B. (1993). Eating disorders and marriage. The couple in focus. New York: Buunner/Mazzel.

Yager, J. (1982). Family issues in the pathogenesis of anorexia nervosa. Psychosomatic Medicine, 44, 43-59 